\title{
Association between circadian rhythms, sleep and cognitive impairment in healthy older adults: an actigraphic study
}

\author{
Andy Cochrane · Ian H. Robertson • \\ Andrew N. Coogan
}

Received: 5 March 2012/ Accepted: 25 March 2012/Published online: 10 April 2012

(C) Springer-Verlag 2012

\begin{abstract}
There is increasing evidence for the relationship between circadian rhythm disturbance and cognitive decline in the older adult. This study measured circadian activity rhythms in a small group of healthy community-dwelling older adults $(n=26)$. Each participant completed a battery of neuropsychological tests and completed sleep diaries and 6 days of actigraphy. Ten participants were identified as having very early signs of cognitive decline as indicated by their performance on the memory tests. Results showed minimal differences on the sleep/activity and circadian parameters across the two groups (declined vs. intact), although there was a significant difference in the acrophase between the declined and intact groups. These findings, although exploratory, suggest that very subtle changes in circadian rhythm may be detected in older adults showing pre-clinical changes in cognitive performance.
\end{abstract}

Keywords Sleep-wake · Circadian · Actigraphy · Older adults $\cdot$ Cognition

\section{Introduction}

The inverse relationship between age and cognitive performance is well recognised. Within this, there is considerable variability across cognitive domains measured and between individuals in the degree and timing of cognitive losses.

\footnotetext{
A. Cochrane · A. N. Coogan ( $ه)$

Department of Psychology, National University of Ireland Maynooth, Maynooth, Co. Kildare, Republic of Ireland

e-mail: andrew.coogan@nuim.ie

I. H. Robertson

Trinity College Institute of Neuroscience, Trinity College,

Dublin 2, Republic of Ireland
}

Cognitive speed, for example, shows a continuous decline from adulthood, whereas crystallised abilities increase up to the sixth to seventh decade (Christensen 2001). Recent evidence suggests that decline across a range of abilities including memory, reasoning and semantic fluency may already be evident in early middle age (45-49 years; SinghManoux et al. 2011). There is increasing evidence for a preclinical stage in dementia in which cognition is borderline compared to normal ageing (Silveri et al. 2007). A recent community-based study identified mild memory impairment in $28 \%$ of a sample of healthy community-dwelling older adults, with deficits in performance not explained by agerelated changes, education levels, mood or health status and suggesting early pathological changes that may eventually progress to mild cognitive impairment (MCI) and dementia (Weaver Cargin et al. 2006). MCI is thought to represent a transitional state between normal ageing and early AD (Grundman et al. 2004), and is diagnosed in those who have an objective and measurable deficit in cognitive functions, but with a preservation of daily activities. The estimates of annual conversion rates to dementia vary across studies but may be as high 10-15\% (Tomaszewski Farias et al. 2009), and thus MCI represents a clinically important stage for identifying individuals at risk.

Circadian rhythms are recurring patterns in a host of physiological, behavioural and cognitive domains that display periods of approximately $24 \mathrm{~h}$ (Reppert and Weaver 2002). It is known that healthy ageing is associated with weakened circadian rhythms, leading to a lessened demarcation between activity in the "day" phase and sleep in the "night" phase, dampened circadian amplitude but unaffected circadian period, phase advances and weakened entrainment to zeitgebers (Hofman and Swaab 2006). As there are circadian rhythms in cognitive domains such as attention, working and episodic memory and executive 
function (Manly et al. 2002; Schmidt et al. 2007), it may be the case that in healthy ageing weakened circadian function is a factor in cognitive decline. In the course of neurodegenerative conditions, such Alzheimer's disease, this deterioration in circadian timekeeping is significantly exacerbated (Thome et al. 2011; Wu and Swaab 2007), and this may contribute to significant behavioural problems such as sundowning (Bachman and Rabins 2006). Aside from the significant circadian dysfunction that is observed in dementia, there is also evidence that age-related cognitive decline in healthy older adults is predicted by the fragmentation of the circadian rhythm in locomotor behaviour (Oosterman et al. 2009). Further evidence that circadian rhythm disturbance may be important in mild cognitive impairment was recently provided by the study of Tranah et al. (2011) who examined actigraphic activity patterns of a large cohort of community-dwelling women in a longitudinal study. These authors report that dampened activity rhythms was a significant predictor for the development of both dementia and MCI, as was a later peak of activity rhythm, suggesting that circadian amplitude and phase may be significant factors in the development of these conditions. Interestingly, these effects were also found to be independent of sleep effects (Tranah et al. (2011)). The role of actigraphically determined daytime activity in MCI and dementia was further examined by Kuhlmei et al. (2011) who report that reduced activity in both dementia and MCI was present when apathy was also present. Factors related to the circadian system, such as overall activity levels and sleep parameters also appear to be important factors contributing to age-related cognitive decline (Blackwell et al. 2011; Barnes et al. 2008). To add to the burgeoning evidence base on circadian rhythm disturbance in age-related cognitive decline we have conducted a study using actigraphy and a neurocognitive battery to examine the relationship between circadian rhythms, sleep, ambulatory activity and cognition in a group of community-dwelling older adults.

\section{Methods}

\section{Participants}

Twenty-six older adults (aged 55 years and over) were recruited from the local community through notices in parish newsletters, libraries and community centres. All participants were living independently, could read and understand English, had normal/corrected vision and MMSE score greater than 24. The study was approved by the National University of Ireland Maynooth ethics committee, and all participants gave written informed consent.
Measurements

\section{Demographic, mood and health variables}

Demographic information was collected related to marital status, and education. Anxiety and depression was assessed using the 7-item anxiety subscale of the Hospital Anxiety and Depression Scale (HADS; Zigmond and Snaith 1983), and the 8-item subset of the Center for Epidemiological Studies-Depression Scale (CES-D; Radloff 1977). Finally, self-reported health was assessed using a single 5-point scale $(1=$ very poor and $5=$ very good $)$.

\section{Neuropsychological assessment}

A battery of tests was administered in one session, and focussed on memory and executive function. The Mini Mental State Exam (MMSE) was used as a screening measure. The National Adult Reading Test (NART-2; Nelson and Willison 1991) was used as a proxy for general intellectual status. Memory was assessed using three subtests of Wechsler Memory Scale: Logical Memory I and II (LM I, LM II: participants recall two paragraphs read aloud by examiner, both immediately and after a delay); Verbal Paired Associates I and II (VPA; examiner presents a list of word pairs, then participant hears one word and must provide the word that went with it); and nonverbal memory measured using Visual Reproduction I and II (VR I and VR II; participants reproduce figures both immediately after presentation and after a delay). Two components of executive functioning were assessed. Semantic fluency was measured by asking participants to name as many animals as possible within 1 min (Gladsjo et al. 1999). Response inhibition was assessed through the Stroop Colour and Word Test (Golden and Freshwater 2002).

\section{Chronotype and subjective sleep measures}

The Morningness-Eveningness Questionnaire (MEQ; Horne and Ostberg 1976) was used to derive a measure of diurnal preference. This consists of 19 items that are summed to yield a score ranging from 16 to 86, with lower values corresponding to evening type (up to 41), scores between 42 and 59 indicating an intermediate phenotype and scores greater than 59 correspond to morning type. Participants maintained a daily sleep diary, from which bedtime, wake-up time, sleep-onset latency and estimated total sleep time was derived.

\section{Actigraphy}

Actigraphy was used to objectively measure daytime activity levels and sleep using Actiwatch 2 (Philips 
Respironics). The device was worn continuously on the participants' non-dominant wrist for 1 week (except for prolonged bathing or swimming). Recordings for six complete days were used for the analysis. Sleep and activity levels were initially calculated using the Actiware 5.59 software. Epoch length was set to $1 \mathrm{~min}$, with a wake threshold value of 40 activity counts. Raw activity data were extracted and analysed in the Chronolab software (Mojón et al. 1992) for analysis of circadian parameters. Data for each subject were averaged over 6 days and analysed by non-linear regression against a $24-\mathrm{h}$ co-sine wave. Data were deemed significantly circadian if the $95 \%$ confidence interval for the amplitude of the rhythm did not include zero. Aside from amplitude, co-sinor analysis revealed values for acrophase (the time of the peak of the fitted $24 \mathrm{~h}$ rhythm) and MESOR, the mean activity level around which the rhythm oscillates. A further measure derived was the percentage of the variance in the activity profile that is explained by the 24-h co-sinor regression (percentage rhythm).

Data analysis

We were interested in subtle symptoms of cognitive decline in this healthy group of older adults. We therefore used the NART-generated IQ score as an estimate of original performance. Z-scores were therefore computed for all of the neuropsychological measures. The older adults were placed in the declined group if there was a difference of over 1SD between the NART and one or more of the Weschler memory subtests used (LMI and II, VRI and II and VPAI and II; Hogan et al. 2012). Otherwise, subjects were placed in the intact group. Groupwise analysis across declined/intact, and morning/intermediate/evening chronotypes was assessed by $t$ tests, one-way between-groups ANOVA and ANCOVA. Correlation analysis was via Pearson product moment correlation or Spearman's rho. Circadian activity profiles between declined and intact groups were assessed by mixed between-within groups ANOVA; $p<0.05$ was deemed significant on all tests.

\section{Results}

Study cohort demographics

The composition of the study participants is shown in Table 1. There were no significant differences across the declined and intact groups in terms of age, sex, HADS, CES-D or subjective rating of general health (Table 1). The declined group performed less well relative to the intact group on the memory subscales (Table 1), but the only
Table 1 Demographic and neuropsychological data for the study cohort

\begin{tabular}{lll}
\hline & Intact $(n=16)$ & Declined $(n=10)$ \\
\hline Age (years) & $71.94(2.53)$ & $70.90(1.5)$ \\
Sex (female:male) & $11: 5$ & $4: 6$ \\
Education (years) & $11.43(0.64)$ & $12.10(1.02)$ \\
Self-report health & $3.94(0.19)$ & $4.20(0.32)$ \\
Depression (CES-D) & $1.19(0.51)$ & $1.20(0.41)$ \\
Anxiety (HADS) & $5.13(0.83)$ & $7.40(1.5)$ \\
Neuropsychological data & & \\
MMSE & $28.00(0.33)$ & $26.90(0.59)$ \\
NART & $109.67(1.76)$ & $111.60(1.4)$ \\
Verbal fluency & $17(1.11)$ & $17.7(1.77)$ \\
Stroop (CWI) & $42.25(1.28)$ & $48.50(3.9)$ \\
LM I & $11.50(0.53)^{* *}$ & $8.30(0.83)$ \\
LMII & $11.50(0.58)^{* *}$ & $8.70(0.78)$ \\
VPA I & $12.27(0.65)$ & $11.89(0.87)$ \\
VPAII & $13.27(0.49)$ & $12.77(0.66)$ \\
VR I & $9.69(0.99)$ & $8.55(0.72)$ \\
VRII & $12.93(0.78)^{*}$ & $10.77(0.64)$
\end{tabular}

Data are presented as means $( \pm$ SEM)

$L M$ logical memory, $V R$ visual reproduction, $V P A$ visual paired associates

* groupwise difference, $p<0.05 ; * * p<0.001$

significant differences that emerged are LM1 $(p=0.002)$ and LM2 $(p=0.008)$ and VR II $(p=0.044)$.

Circadian parameters

When groupwise comparisons of study subjects' chronometrics were carried out, a significant difference in the acrophase between the declined and intact groups $(15.16 \pm 0.23 \mathrm{~h}$ for the declined vs. $14.15 \pm 0.32 \mathrm{~h}$ for the intact group, $p<0.05$; Fig. 1) was found. The significant groupwise difference in acrophase persists after controlling for age, health, HADS, CES-D or sex. There was no difference between the declined and intact groups for amplitude, MESOR or percentage of variance in activity explained by the 24-h co-sine wave (Fig. 1). Mixed between-within groups ANOVA analysis of the mean 24-h activity profiles in the declined and intact groups showed a main effect of time $\left(F_{47,1,034}=20.18, p<0.001\right)$, but no time $\times$ group interaction nor a main effect of group ( $p=0.92$ and $p=0.112$, respectively; Fig. 2). There were no significant correlations between any of chronometrics examined and the various cognitive scores used. There was also no difference between the declined and intact groups on their MEQ scores $(58.33 \pm 2.93$ vs. $57.27 \pm 2.34$, respectively, $p=0.78$ ). Chi-square analysis for cognitive 

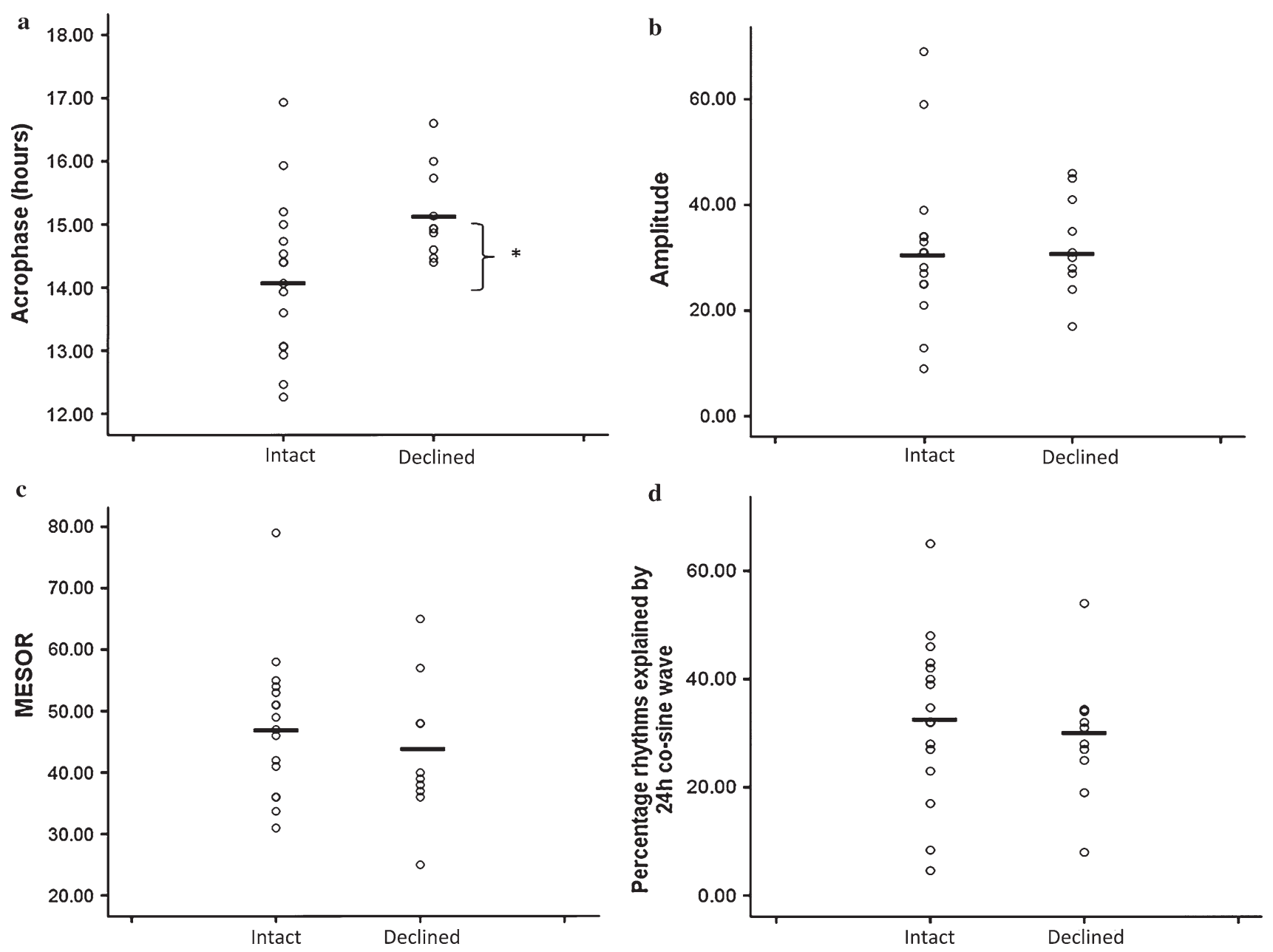

Fig. 1 Scatterplots showing the circadian chronometrics (a acrophase, $\mathbf{b}$ amplitude, $\mathbf{c}$ MESOR and $\mathbf{d}$ percentage variance in activity pattern explained by the 24-h co-sinor regression model) for study

group distribution across the chronotypes also did not reveal any significant relationship $\left(\chi^{2}=0.152, p=0.92\right)$.

\section{Activity and sleep}

The objective and self-reported activity and sleep data are presented in Table 2. Groupwise comparisons between the declined and intact group revealed no significant difference in the total activity or the duration of the active period (there was also no significant differences in activity across chronotypes). Correlation analysis did not reveal any significant relationships between total activity or activity duration and any of the cognitive measures examined. When groupwise comparisons were undertaken for objective and self-reported sleep parameters between the declined and intact groups, there were no significant differences in any of the parameters examined (Table 2). Correlation analysis between cognitive measures (Stroop, MMSE, NART, LM 1 and 2, VPA 1 and 2, and VR 1 and

participants in the intact and cognitively declined groups. The horizontal lines indicate the mean value for each group. ${ }^{*} p<0.05$ between groups

2) and sleep parameters, whilst controlling for age, HADS, CES-D and subjective general health scores, revealed significant inverse correlations between wake after sleep onset (WASO) and Stroop $(r=-0.538, p<0.05)$ and between LM1 and sleep duration $(r=-0.539, p<0.05)$.

\section{Discussion}

This study has examined the relationship of circadian rhythms, sleep and cognitive function in healthy older adults. Actigraphy allows for the subjective evaluation of gross patterns in activity and the derivation of objective sleep parameters, whilst being minimally intrusive for study participants (compared to polysomnography, for example). Its combination with neuropsychological test batteries allows for the investigation of links between rhythms, sleep and cognition, without having to rely on subjective self-report methodology. Examining such links 


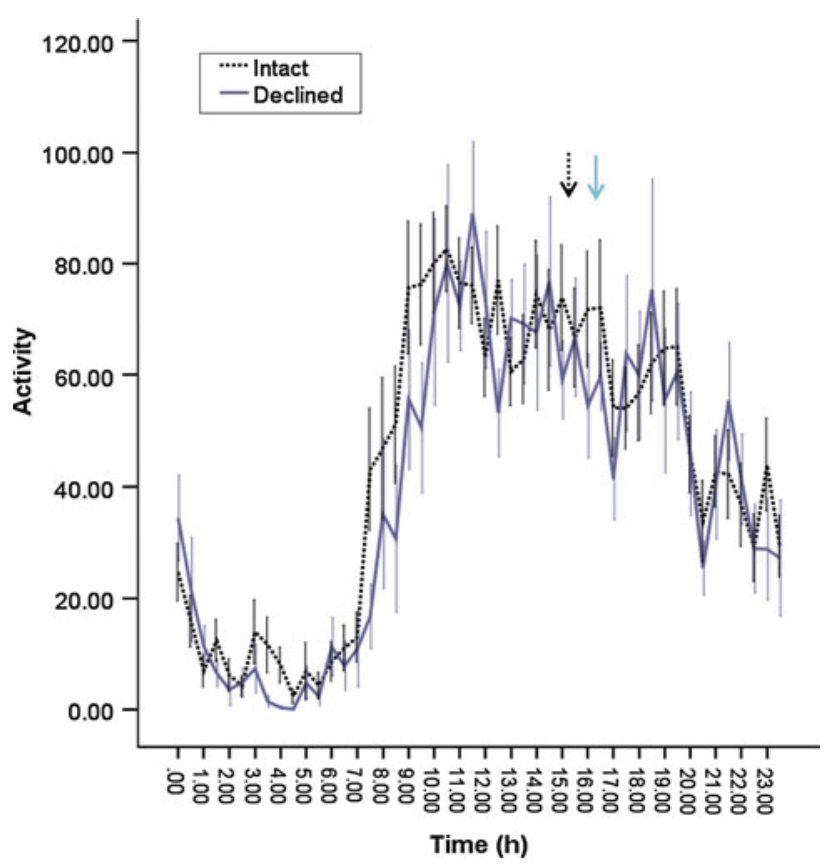

Fig. 2 Average 24-h activity profiles for the intact (dotted line) and declined (continuous line) groups. The dotted arrow indicates the acrophase for the intact group, and the continuous lined arrow that for the declined group. Data are mean \pm SEM

Table 2 Actigraphic data for sleep and circadian measures, as well as self-report data for sleep and diurnal preference

\begin{tabular}{lll}
\hline & Intact $(n=16)$ & Declined $(n=10)$ \\
\hline Actigraphy & & \\
Activity duration (min) & $876: 79(21.37)$ & $874: 97(21: 02)$ \\
Activity counts/min & $252.53(70.59)$ & $246.78(31.50)$ \\
Total sleep time (min) & $415: 30(63: 88)$ & $455: 37(87: 94)$ \\
Sleep time & $00: 12(0: 17)$ & $00: 13(0: 16)$ \\
Wake time & $07: 43(0: 19)$ & $08: 09(0: 18)$ \\
Sleep efficiency & $84.11 \%(8.72)$ & $82.90 \%(6.71)$ \\
WASO & $36.77(25.21)$ & $30.93(11.88)$ \\
Percentage rhythm & $33.50(15.44)$ & $24.85(18.16)$ \\
MESOR & $47.81(11.47)$ & $42.90(11.41)$ \\
Amplitude & $32.64(14.17)$ & $30.68(10.05)$ \\
Acrophase & $14: 16(0: 32) *$ & $15: 17(0: 23)$ \\
Self-report & & \\
Total MEQ & $57.27(2.34)$ & $58.33(2.93)$ \\
Sleep diary bed time & $23: 52$ & $23: 32$ \\
Sleep latency (min) & $26: 51(5.17)$ & $33: 22(15.48)$ \\
Total sleep time (min) & $401: 33(718.54)$ & $433: 13(38.08)$ \\
\hline
\end{tabular}

Data are presented as means $( \pm$ SEM)

$M E Q$ morning-evening questionnaire score, WASO wake after sleep onset

* groupwise difference, $p<0.05$ is likely to be of considerable importance given both the high prevalence of MCI and the significant age-related disruption of the circadian timing system (Weaver Cargin et al. 2006; Hofman and Swaab 2006). Further, the examination of sleep in age-related cognitive impairment may yield important insight, given that the cognitive roles of sleep and the impairments that are attendant on sleep disruption appear to change with age (Pace-Schott and Spencer 2011). To date, there have only been relatively few studies examining measures of sleep in age-related cognitive decline and $\mathrm{MCI}$ indicating the need for further and more focussed examination of these issues (BeaulieuBonneau and Hudon 2009).

Our analysis of the circadian rhythm in gross behaviour shows that there are not significant differences between the declined and the intact groups in terms of the rhythm amplitude, MESOR or percentage of variance in the activity rhythm explained by the 24 -h co-sinor regression model, but that those in the declined group showed a peak in their activity rhythm that was significantly later than that for the intact group. This finding appears to be in agreement with the recent report from Tranah et al. (2011), who in a large prospective study found that delayed behavioural rhythm was a significant predictor of developing MCI or dementia. However, these authors also report that dampened circadian amplitude and robustness of the rhythm were also predictors of $\mathrm{MCI} / \mathrm{dementia}$, findings that are not recapitulated in our study. However, given the modest sample size in the current study and the mild nature of the cognitive decline used to classify participants in our study, it is not necessarily surprising to note such discrepancies. Further, the study of Tranah et al. (2011) was exclusively in women, and a sex-specific effect cannot be ruled out. Interestingly, in the current study the delay in acrophase was not affected by including possible confounders in the statistical analysis (e.g. age, depression ratings), but also did not seem to translate either into differences in the MEQ scores for subjective ratings of diurnal preference, nor into altered objectively determined sleep and wake times, indicating that changes in circadian timing may not be translated into changes in sleep parameters in this case. This finding seems to be congruent with the finding that increased risk of dementia and MCI reported by Tranah et al. (2011) associated with altered circadian rhythms was also not altered after adjusting for sleep efficiency (which in our study was not different between groups), suggesting that it is the circadian rhythm alteration, and not a secondary effect of such on sleep, that may be of primary importance in the development of MCI or dementia.

Our finding that there were no differences in overall activity levels between the declined and intact group may 
be explained by the fact that we did not assess apathy, as recently Kuhlmei et al. (2011) have shown that daytime activity is lower in MCI, but only when apathy is present. As apathy is a common finding in MCI and dementia patients, future studies will need to take cognisance of this in their design. Our study also provides data on objectively determined sleep parameters in cognitively declined older adults. There have been a number of reports of subjectively determined sleep problems in MCI, with prevalence of sleep disturbance ranging from 14 to $59 \%$ (BeaulieuBonneau and Hudon 2009). Studies that have compared sleep disturbances in MCI to healthy controls and to dementia patients have reported that the rate of sleep disturbance in MCI is approximately double that in controls, but half that in dementia (Geda et al. 2004). However, given the potential for disparity between objective and subjective sleep measures, there is a clear requirement for the objective assessment of sleep in age-related cognitive decline, and some recent studies have addressed the links between sleep architecture, sleep disordered breathing and MCI (Yaffe et al. 2011; Blackwell et al. 2011). In situations where polysomnography is not available or practical, actigraphy can provide valuable objective sleep data (Ancoli-Israel et al. 2003). In our current results we report no differences in any sleep measure between the declined and intact groups. Both groups showed very similar going to bed and waking times, showed equivalent levels of sleep efficiency and wake after sleep onset, and did not differ in terms of the sleep bout duration. Therefore, differences in cognition observed between the two groups do not seem to be explained by alterations in gross measures of sleep. Future studies may address themselves to the use of polysomnography to determine sleep architecture in cognitively declined older adults, and may reveal differences in sleep staging that are inscrutable in the current study.

There are a number of weaknesses to be considered in the current study: first is the relatively small sample size in this exploratory study; second is that participants did not carry a clinical diagnosis of MCI, rather they were placed into the declined/intact groups according to their performance on the neuropsychological test battery normalised to NART scores. Thus, while we were able to categorise a small subgroup as showing some decline in performance, the degree of deficit may have been relatively minor and this may account for the limited differences between the two groups on the sleep and circadian parameters, and more pronounced differences may be observed for those with clinical diagnoses of MCI. A further limitation of the current study is that no biological measures of circadian function were assayed. Work in pre-clinical models has suggested that healthy ageing is associated with alterations in the molecular components of the circadian clock (e.g. Wyse and Coogan 2010) and such alterations may be pertinent to the issue of circadian rhythms and cognition in healthy older adults.

In conclusion, the present study adds further evidence that alterations in circadian timekeeping may be of importance in the development of age-related cognitive decline in healthy adults. Future studies may further address the nature of such circadian deficits-for example, is the delayed acrophase of the rhythm observed here a consequence of altered entrainment to appropriate environmental zeitgebers, an alteration in core clock processes, or secondary to other behavioural changes that accompany age-related cognitive decline?

Acknowledgments We acknowledge support from the Trinity College Dublin Arts and Social Sciences Benefaction Fund.

\section{References}

Ancoli-Israel S, Cole R, Alessi C, Chambers M, Moorcroft W, Pollak CP (2003) The role of actigraphy in the study of sleep and circadian rhythms. Sleep 26:342-392

Bachman D, Rabins P (2006) "Sundowning" and other temporally associated agitation states in dementia patients. Annu Rev Med 57:499-511

Barnes DE, Blackwell T, Stone KL, Goldman SE, Hillier T, Yaffe K (2008) Cognition in older women: the importance of daytime movement. J Am Geriatr Soc 56:1658-1664

Beaulieu-Bonneau S, Hudon C (2009) Sleep disturbances in older adults with mild cognitive impairment. Int Psychogeriatr 24:654-666

Blackwell T, Yaffe K, Ancoli-Israel S, Redline S, Ensrud KE, Stefanick ML, Laffan A, Stone KL, Osteoporotic Fractures in Men Study Group (2011) Associations between sleep architecture and sleep-disordered breathing and cognition in older community-dwelling men: the Osteoporotic Fractures in Men Sleep Study. J Am Geriatr Soc 59:2217-2225. doi:10.1111/ j.1532-5415.2011.03731

Christensen H (2001) What cognitive changes can be expected with normal ageing? Austral NZ J Psychiat 35:768-775

Geda YE, Smith GE, Knopman DS, Boeve BF, Tangalos EG, Ivnik RJ, Mrazek DA, Edland SD, Petersen RC (2004) De novo genesis of neuropsychiatric symptoms in mild cognitive impairment (MCI). Int Psychogeriatr 16:51-60

Gladsjo JA, Schumam CC, Evans JD, Peavy GM, Miller SW, Heaton RK (1999) Norms for letter and category fluency : demographic corrections for age, education, and ethnicity. Assessment 6:147-178

Golden CJ, Freshwater SM (2002) Stroop colour and word test: revised examiner's manual. Stoelting, Wood Dale

Grundman M, Petersen RC, Ferris SH, Thomas RG, Aisen PS, Bennett DA, Foster NL, Jack CR Jr, Galasko DR, Doody R, Kaye J, Sano M, Mohs R, Gauthier S, Kim HT, Jin S, Schultz AN, Schafer K, Mulnard R, van Dyck CH, Mintzer J, Zamrini EY, Cahn-Weiner D, Thal LJ, Alzheimer's Disease Cooperative Study (2004) Mild cognitive impairment can be distinguished from Alzheimer's disease and normal ageing for clinical trials. Arch Neurol 61:59-66

Hofman MA, Swaab DF (2006) Living by the clock: the circadian pacemaker in older people. Ageing Res Rev 5:33-51

Hogan MJ, Kenney JPM, Roche RAP, Keane M, Moore JL, Kaiser J (2012) Behavioural and electrophysiological effects of visual paired associate context manipulations during encoding and 
recognition in younger adults, older adults and older cognitively declined adults. Exp Brain Res 216:241-250

Horne JA, Ostberg O (1976) A self-assessment questionnaire to determine morningness-eveningness in human circadian rhythms. Intl J Chronbiol 4:97-110

Kuhlmei A, Walther B, Becker T, Muller U, Nikolaus T (2011) Actigraphic daytime activity is reduced in patients with cognitive impairment and apathy. Eur Psychiatry (E-Pub June 2011)

Manly T, Lewis GH, Robertson IH, Watson PC, Datta AK (2002) Coffee in the cornflakes: time-of-day as a modulator of executive response control. Neuropsychologia. 40:1-6

Mojón A, Fernández JR, Hermida RC (1992) Chronolab: an interactive software package for chronobiologic time series analysis written for the Macintosh computer. Chronobiol Int 9:403-412

Nelson HE, Willison J (1991) National Adult Reading Test (NART): test manual, 2nd edn. NFER Nelson, Windsor

Oosterman JM, van Someren EJ, Vogels RL, Van Harten B, Scherder EJ (2009) Fragmentation of the rest-activity rhythm correlates with age-related cognitive deficits. J Sleep Res 18:129-135

Pace-Schott EF, Spencer RM (2011) Age-related changes in the cognitive function of sleep. Prog Brain Res 191:75-89

Radloff LS (1977) The CES-D scale: a self-report depression scale for research in the general population. Appl Psychol Meas $1: 385-401$

Reppert SM, Weaver DR (2002) Coordination of circadian timing in mammals. Nature 418:935-941

Schmidt C, Collette F, Cajochen C, Peigneux P (2007) A time to think: circadian rhythms in human cognition. Cogn Neuropsychol 24:755-789

Silveri MC, Reali G, Jenner C, Puopolo M (2007) Attention and memory in the preclinical stage of dementia. J Geriatr Psychiatry Neurol 20:67-75
Singh-Manoux A, Kivimaki M, Glymour MM, Elbaz A, Berr C, Ebmeier KP, Ferrie JE, Dugravot A (2011) Timing of onset of cognitive decline: results from Whitehall II prospective cohort study. Brit Med J 344:d7622. doi:10.1136/bmj.d7622

Thome J, Coogan AN, Woods AG, Darie CC, Häßler F (2011) CLOCK genes and circadian rhythmicity in Alzheimer disease. J Aging Res 2011:383091

Tomaszewski Farias ST, Mungas D, Reed BR, Harvey D, DeCarli C (2009) Progression of mild cognitive impairment to dementia in clinic- vs community-based cohorts. Arch Neurol 66:1151-1157

Tranah GJ, Blackwell T, Stone KL, Ancoli-Israel S, Paudel ML, Ensrud KE, Cauley JA, Redline S, Hillier TA, Cummings SR, Yaffe K, SOF Research Group (2011) Circadian activity rhythms and risk of incident dementia and mild cognitive impairment in older women. Ann Neurol 70(722):732

Weaver Cargin J, Maruff P, Collie A, Masters C (2006) Mild memory impairment in healthy older adults is distinct from normal ageing. Brain Cogn 60:146-155

Wu YH, Swaab DF (2007) Disturbance and strategies for reactivation of the circadian rhythm system in aging and Alzheimer's disease. Sleep Med 8:623-636

Wyse CA, Coogan AN (2010) Impact of aging on diurnal expression patterns of CLOCK and BMAL1 in the mouse brain. Brain Res 1337:21-31

Yaffe K, Laffan AM, Harrison SL, Redline S, Spira AP, Ensrud KE, Ancoli-Israel S, Stone KL (2011) Sleep-disordered breathing, hypoxia, and risk of mild cognitive impairment and dementia in older women. JAMA 306:613-619

Zigmond AS, Snaith RP (1983) The Hospital Anxiety and Depression Scale. Acta Psychiatr Scand 67:361-370 